\title{
Contribuição da etnoictiologia à análise da legislação pesqueira referente ao defeso de espécies de peixes de interesse comercial no oeste da Amazônia Brasileira, rio Guaporé, Rondônia, Brazil
}

\author{
Carolina Rodrigues da Costa Doria ${ }^{1 *}$ \\ Túlio Raimundo de Araújo ${ }^{1}$ \\ Suelen Taciane Brasil de Souza ${ }^{2}$ \\ Gislene Torrente-Vilara ${ }^{3}$ \\ ${ }^{1}$ Departamento de Biologia, Laboratório de Ictiologia e Pesca \\ Universidade Federal de Rondônia/UNIR \\ BR 364, Km 9,5 CEP: 78900-000, Porto Velho - RO, Brasil \\ ${ }^{2}$ Ação Ecológica Guaporé - ECOPORÉ, Costa Marques - RO, Brasil \\ ${ }^{3}$ Programa de Pós-Graduação em Biologia Tropical e Recursos Naturais \\ Instituto Nacional de Pesquisas da Amazônia/UFAM, Manaus - AM, Brasil \\ vilara@uol.com.br \\ *Autor para correspondência \\ carolinarcdoria@uol.com.br
}

Submetido em 11/06/2007

Aceito para publicação em 10/01/2008

\section{Resumo}

Conflitos pesqueiros têm sido observados na região do Guaporé entre pescadores e órgãos gestores quanto ao período oficial de defeso reprodutivo. Os pescadores afirmam que o defeso não corresponde à época de reprodução das principais espécies comercializadas, o que pode estar prejudicando os estoques locais de peixes. Este trabalho comparou o conhecimento ecológico tradicional (CET) com informações obtidas em pescarias experimentais e informação obtida da literatura científica sobre os períodos de reprodução das principais categorias de pescado desembarcadas naquela região. Das 28 categorias de pescado analisadas, 14 (50\%) foram capturadas na pesca experimental e puderam ser avaliadas. O conhecimento ecológico tradicional confirmou a informação experimental para 10 categorias de pescado (72\%). Os resultados sugerem a necessidade de ajustes no período oficial de defeso para as categorias: caparari (Pseudoplatystoma tigrinun), surubim (P. fasciatum), tambaqui (Colossoma macropomum), pescada (Plagioscion spp) e tucunaré (Cichla spp). A discussão aborda a possível inadequação do período de defeso estabelecido com bases em informações geradas em diferentes bacias e aplicados para grandes áreas da Amazônia brasileira. Este estudo confirma o refinado conhecimento do pescador sobre a biologia das espécies que ele explora e sugere que este CET pode ser utilizado para ajustar políticas de gestão pesqueira regionalmente.

Unitermos: categorias de pescado, conhecimento ecológico tradicional, biologia reprodutiva, período de defeso, conflitos pesqueiros 


\section{Abstract}

Ethnoichthyological contribution to the official fisheries document concerning fisheries closure of some commercial fish categories in the western Brazilian Amazon, Guaporé River, Rondônia, Brazil. There are conflicts among fishermen and local environmental protection agencies that regulate fishing in the area, concerning the official closure periods of the fisheries. The fishermen affirm that the dates established for protection of spawning do not correspond to the spawning season of the primary commercialized species, and that this could be hindering the local fish markets. This report compares the traditional ecological knowledge (TEK) with information obtained from experimental fishery and scientific data covering the reproduction periods of the primary categories of fish market in the region. Of the 28 fish categories analyzed, 14 (50\%) were captured in experimental fishing and were evaluated. The TEK confirmed the experimental information for 10 categories of fish $(72 \%)$. The results suggest the necessity of adjusting the official protection dates stipulated for the following fish categories: caparari (Pseudoplatystoma tigrinun), Sorubim (P. fasciatum), tambaqui (Colossoma macropomum), pescada (Plagioscion squamosissimus) and tucunaré (Cichla ocellaris). The discussion deals with a possibly inadequate period of protection based on the information obtained from different basins applied to larger and more diverse areas of the Brazilian Amazon Rainforest. This study confirms the refined biological knowledge that the fishermen have of the species they exploit and suggests that the traditional ecological knowledge can be useful to adjust political issues dealing with the regional protection agency of fishing.

Key words: fish categories, traditional ecological knowledge, reproductive biology, fish protection period, fisheries conflicts

\section{Introdução}

A pesca é tradicionalmente a atividade extrativista mais importante da Bacia Amazônica. O peixe é a principal fonte de proteína animal regional e garante recursos para a maioria das populações tradicionais da Amazônia (Smith, 1979).

Caracteristicamente multiaparelhada e multiespecífica (Batista, 1998), a tradição da pescaria incorporou o conhecimento ecológico tradicional do pescador, tornando-a uma arte. A distribuição e a abundância das espécies de peixes ao longo dos rios, várzeas e igapós é influenciada pela variação sazonal das águas (Junk et al., 1989). O apurado conhecimento sobre a dinâmica das águas e o movimento dos peixes em função do alagamento sazonal das florestas permite ao pescador experiente selecionar os locais para a pesca e os aparelhos mais eficientes na captura de cada espécie, em cada fase do ciclo hidrológico (Merona e Gascuel, 1993). A experiência do pescador não está limitada ao conhecimento da distribuição, rotas migratórias e épocas de capturas abundantes de cada espécie. Um refinado conhecimento dos ecossistemas tem sido demonstrado por pescadores em relação às espécies de peixes com peculiaridades sobre territorialidade, espectro alimentar, áreas de alimentação e atividade reprodutiva (Morril, 1967; Ankei, 1982, Begossi e
Garavello, 1990; Begossi e Figueiredo, 1995; Silvano e Begossi, 2002, Batistella et al., 2005).

O conhecimento ecológico tradicional (CET) é definido na literatura como o conhecimento acumulado a respeito das espécies, do ambiente e suas interações e repassado de geração para geração (Drew, 2005). A etnociência estuda este conhecimento das populações humanas sobre o funcionamento dos processos naturais, na tentativa de descobrir a lógica subjacente ao conhecimento humano sobre o mundo natural (Diegues, 2000).

O conhecimento ecológico tradicional (CET) tem sido abordado como importante fonte de informação para conservação de recursos naturais (Diegues, 2000; Silvano e Begossi, 2002; Drew, 2005). Estudos recentes nesta área reconhecem o importante papel das comunidades tradicionais nesse sentido, incorporando esse conhecimento ao conjunto de informações técnico - científicas disponível para subsidiar as políticas públicas conservacionistas e o manejo pesqueiro regional (Diegues, 2000; Castro, 2000; Costa-Neto et al., 2002; Silvano e Begossi, 2002; Batista et al., 2004; Drew, 2005; Berkers et al., 2006). O ramo da etnociência que estuda o conhecimento tradicional sobre o pescado é a etnoictiologia e tem o objetivo de compreender o fenômeno da interação da espécie humana com os recursos ícticos, englobando aspectos tanto cognitivos do 
homem em relação ao comportamento do peixe quanto do seu próprio comportamento em relação ao pescado (Marques, 1995).

Existem diversas estratégias tradicionais de manejo da pesca aplicadas pela população ribeirinha e a efetividade desse processo foi observada e avaliada em alguns trabalhos (Batista, 1998; McGrath et al., 1994; Issac e Cerdeira, 2004). Estes trabalhos reconhecem a possibilidade de obter sucesso no manejo e ordenamento pesqueiro a partir de conhecimento ecológico tradicional e, por sua vez, abrem espaços de negociação entre os atores, permitindo o surgimento de políticas com melhores chances de sustentabilidade quando consideram o conhecimento tradicional, complementado pelo conhecimento científico (Azevedo e Apel, 2004; Berkers et al., 2006). Um dos efeitos positivos dessas negociações é a diminuição dos conflitos da atividade pesqueira entre os diversos atores e o governo.

Um exemplo claro de situações de conflito pode ser observado na contestação dos pescadores profissionais sobre legislação da pesca no estado de Rondônia. De acordo com a percepção dos pescadores da região do Guaporé, o período de defeso estabelecido pelos órgãos ambientais gestores da pesca não tem correspondido com a época de reprodução das principais categorias comercializadas (tambaqui, pescada, tucunaré e surubim/caparari) o que poderia comprometer os estoques locais (Doria et al., 2004).

Dentro deste contexto, visando subsidiar discussões sobre o ordenamento pesqueiro na bacia do Guaporé, o presente trabalho teve como objetivo investigar se: 1 . O conhecimento ecológico tradicional do pescador sobre o período reprodutivo das categorias de pescado é confirmado pela pescaria experimental. 2. O período reprodutivo apontado pelo CET dos pescadores confere com a legislação sobre o defeso de cada categoria de pescado. 3. O período de defeso para as categorias de pescado estabelecido pela legislação da pesca aplicada na região do rio Guaporé é confirmado pela pescaria experimental.

\section{A área de estudo}

A sub-bacia do rio Guaporé foi escolhida como área de estudo por representar um dos principais trechos de pesca do estado de Rondônia. Atuam na região pescadores ligados às colônias de pescadores dos municípios de Guajará Mirim, Costa Marques e Pimenteiras. Estima-se uma produção média de pescado local de 350 toneladas (para os anos de 2002 e 2003) na porção brasileira, envolvendo cerca de 150 pescadores profissionais (Doria et al., 2004).

A Colônia de Pescadores de Costa Marques foi selecionada como ponto representativo para obtenção das informações etnoictiológicas em função de seus pescadores terem gerado demandas de investigação nesse sentido, relatadas em outros estudos sobre a pesca e ordenamento pesqueiro na região. Esses estudos antecedentes foram realizados pela mesma equipe do Laboratório de Ictiologia e Pesca da Universidade Federal de Rondônia e ECOPORÉ, desde fevereiro de 2002 e estão em formato de relatórios técnicos. Este fato garantiu o acesso aos entrevistados e orientou esse trabalho no sentido de reunir mais informações sobre a pesca naquela localidade.

Os dados biológicos das populações de peixes foram obtidos em pescarias experimentais no rio Cautário afluente do rio Guaporé (Figura 1). A sub-bacia do Guaporé abrange aproximadamente 700 mil hectares, situados entre S $12^{\circ} 35^{\prime}$ e $13^{\circ} 30^{\prime}$ e W $60^{\circ} 55^{\prime}$ e $64^{\circ} 20$. Ao sul dessa região está o chaco boliviano, com extensos campos inundáveis e formações aluviais, entremeados por grandes lagos. Ao norte, ocorre a floresta amazônica de terra firme, floresta ombrófila, e a leste, a faixa de transição entre os biomas da Amazônia e do Cerrado (Fernandes e Guimarães, 2002). O rio Cautário está localizado no nordeste da sub-bacia do Guaporé e é um dos seus principais afluentes, drenando principalmente o município de Costa Marques, Rondônia. É um rio meândrico com águas claras e profundidade não ultrapassando 2-3 metros na seca e cerca de 5 a 6 metros durante a curta estação da cheia local. O substrato é constituído por areia e durante a enchente, a baixa planície de inundação com barrancos que não ultrapassam três metros em secas extremas, inundam uma extensa área de floresta adentro. A vegetação é principalmente composta por floresta ombrófila aberta (63\%) (Olmos et al., 1998) e manchas de cerrado, com formação de espessos tapetes alagáveis de gramíneas e ciperáceas, em menor proporção quando comparado a extensão 


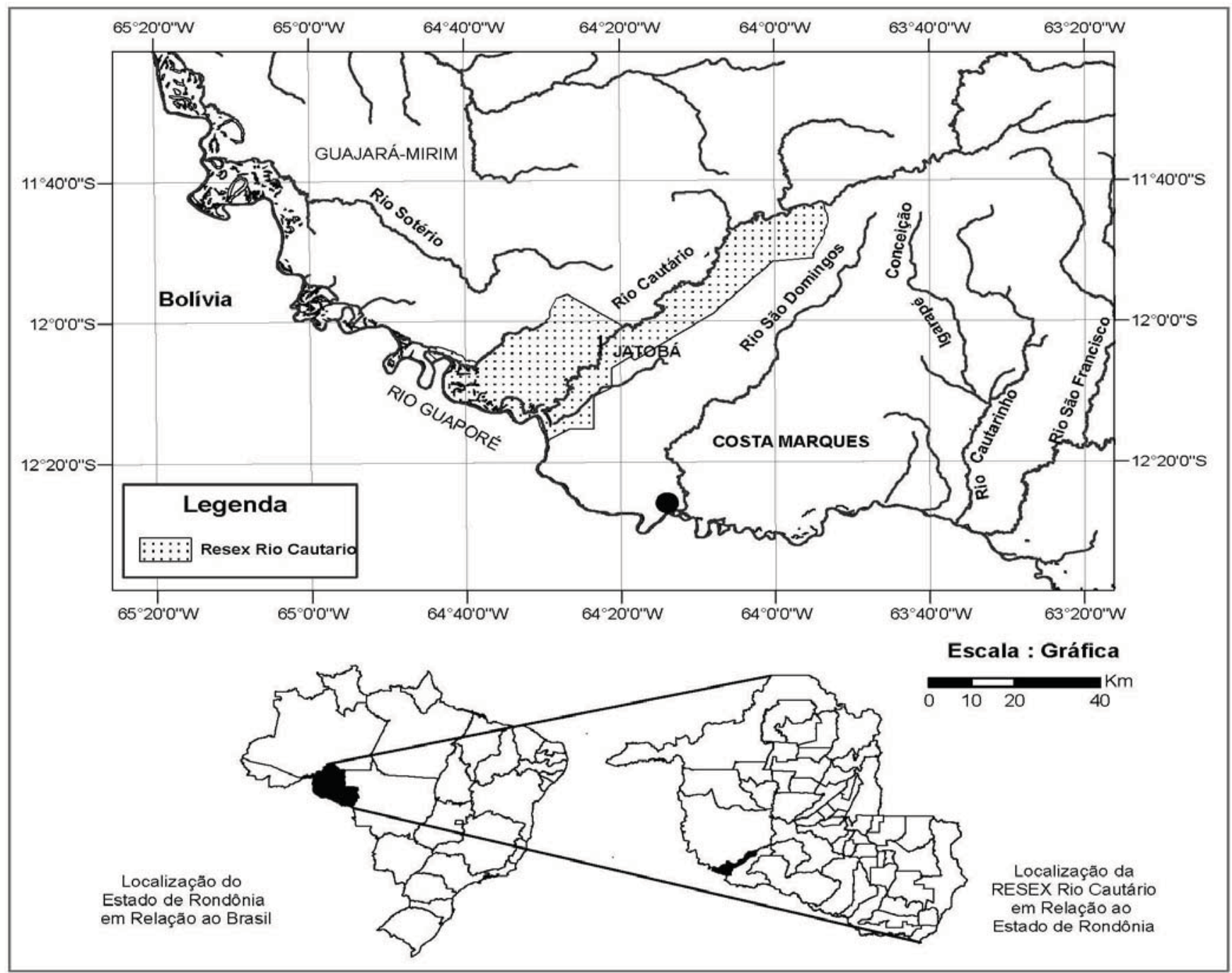

FIGURA 1: Área de estudo, delimitando a Reserva Extrativista Estadual do Rio Cautário e indicando a Colocação Jatobá e o município de Costa Marques.

dessas manchas encontradas nas demais áreas ao longo da calha do rio principal, o rio Guaporé.

O rio Cautário recorta a Reserva Extrativista (RESEX) Estadual e a RESEX Federal do rio Cautário. As Reservas Extrativistas são unidades de conservação, destinadas a exploração sustentável e a conservação dos recursos naturais renováveis, por populações com tradição no uso de recursos extrativos (Allegretti, 1987). A RESEX Estadual do rio Cautário tem uma população estimada em 282 moradores (únicos usuários da área), que vive de atividades extrativistas e de subsistência. Abrange a porção médio-baixa do rio Cautário, numa área de $144.371,657$ ha e pertencente ao município de
Costa Marques. As áreas alteradas pelo uso humano ocupam apenas 6,5\% da reserva (Olmos et al., 1998), apresentando alto grau de conservação nas porções próximas aos cursos d'água. A atividade pesqueira na área é realizada pelos comunitários somente para a subsistência, estando em fase de estudos o manejo comunitário do pescado para comercialização, de acordo com a normatização pertinente (SNUC, 2002).

O ponto de coleta selecionado da RESEX Estadual do Rio Cautário é denominado na região como Colocação Jatobá (S 1203’21.9” W 64²2’33.5”). 


\section{Material e Métodos}

\section{Dados etnoictiológicos}

As informações foram coletadas durante entrevistas individuais nos dias 10 de maio, 15 e 16 de agosto de 2005, com 31 (63\%) pescadores profissionais da Colônia de Pescadores de Costa Marques (Z-4), do total de 49 pescadores registrados e ativos da referida colônia. Para a seleção dos entrevistados, foram considerados os pescadores mais "experientes", aqueles com no mínimo 15 anos de atividade pesqueira, e o interesse pessoal de cada um em responder as questões após breve explanação da pesquisa. As entrevistas foram realizadas na colônia de pescadores e no porto pesqueiro por um biólogo da equipe, com duração de cerca de 15 a 20 minutos.

A composição da lista de categorias de pescado a serem analisadas considerou os registros do estudo de dinâmica da pesca no município de Costa Marques ${ }^{1}$ (Brasil de Souza et al. 2004), sobre as principais categorias capturadas na região, utilizando a classificação tradicional dos pescadores, a identificação taxonômica e a lista das espécies analisadas nas pescarias experimentais, totalizando uma lista com 28 categorias de pescado (Tabela 2). O roteiro de perguntas aplicado para avaliar o conhecimento ecológico tradicional era composto de questões abertas sobre:

- o período em que a categoria encontra-se "ovada", no qual, segundo os pescadores, 1. as "ovas" saem facilmente; 2. o peixe apresenta "ovas grandes na barrigada" ou; 3. a "barrigada cheia de ova". Essas características conferem com o estádio de maturação avançada (maduro) na escala determinada por Vazzoler (1996), com ovários desenvolvidos, ovócitos maduros, ocupando quase toda a parte interna da cavidade celomática, com grandes vasos sanguíneos e de fácil extrusão;

- os principais conflitos na percepção dos pescadores entre a legislação do defeso e a proteção efetiva do período reprodutivo das categorias de pescado.
Em ambos os casos, o período reprodutivo informado por $80 \%$ ou mais dos entrevistados que responderam às perguntas foi considerado como período determinado pelo conhecimento ecológico tradicional (CET).

\section{Dados experimentais}

A escolha do ponto para as pescarias experimentais foi baseada nos resultados do questionário sócio-econômico-ambiental ${ }^{2}$, aplicado em 29 famílias da comunidade da reserva, nos dias 7 e 8 de março de 2003. O objetivo deste questionário foi orientar a escolha do ponto mais favorável a captura em relação à diversidade de "qualidade de pescado" conforme a percepção da comunidade local. Desta forma, o resultado do questionário apontou uma área que engloba maior número de espécies representativas das categorias desembarcadas no mercado de Costa Marques.

As pescarias experimentais foram realizadas bimestralmente, entre março de 2003 e março de 2004. Foram utilizados dois conjuntos de malhadeiras, cada um composto por 10 diferentes malhas $(30,40,50,60$, 70, 80, 90, 100, 110 e 120mm entre-nós opostos), com área conhecida e padronizada. As malhadeiras eram armadas por um período de 24 horas, associadas à captura por espinhel.

Os exemplares capturados foram imediatamente transportados para um laboratório de campo para identificação taxonômica e biometria, obtendo-se o comprimento padrão $(\mathrm{mm})$ e o peso total $(\mathrm{g})$. Posteriormente foi realizada uma incisão abdominal em cada exemplar para identificação macroscópica do sexo e, para fêmeas, observou-se o estádio de desenvolvimento gonadal (Vazzoler, 1996).

Para capturar os exemplares jovens das espécies comerciais, comumente não capturadas com malhadeiras, padronizou-se três lances consecutivos com rede de cerco (malha $6 \mathrm{~mm}$ e dimensão 5 X 10m), com área de captura estimada em $100 \mathrm{~m}^{2}$, utilizada nas macrófitas

1 O estudo da Dinâmica da pesca no município de Costa Marques é um componente do Projeto Manejo Comunitário da Pesca em RESEXs do Guaporé, desenvolvido pela Associação dos Seringueiros do Guaporé com apoio técnico da organização não governamental ECOPORÉ e do Laboratório de Ictiologia e Pesca - Universidade Federal de Rondônia, no período de Fevereiro de 2002 até Fevereiro 2007.

2 Este questionário foi aplicado pela equipe do Projeto Manejo Comunitário da Pesca em RESEXs do Guaporé. 
aquáticas e praias, conforme disponibilidade de ambiente em cada período hidrológico. Exemplares representativos das espécies capturadas foram depositados na coleção de peixes do Instituto Nacional de Pesquisas da Amazônia (INPA) sob a numeração 21675 a 21911.

A análise dos dados experimentais considerou como período reprodutivo os meses com maiores freqüências de indivíduos em maturação avançada (maduros) e em reprodução (desovando), reunindo as espécies conforme cada categoria de pescado identificada pelos pescadores. A Freqüência Reprodutiva (FR), freqüência de exemplares capturados nos estádios "maduro" e "em reprodução" para o total das amostras (percentual) foi obtida para cada categoria. Posteriormente, o período reprodutivo determinado a partir dos dados experimentais foi comparado com as informações obtidas pelo CET, com a legislação pertinente e com informações científicas secundárias obtidas na literatura, sempre respeitando as respectivas categorias do pescado. Os resultados foram apresentados em formato de tabelas comparando as situações descritas acima.

A autorização de pesquisa foi obtida junto a Associação dos Seringueiros do Vale do Guaporé, responsável pela gestão da RESEX Estadual do Rio Cautário, Secretaria Estadual de Desenvolvimento e Meio Ambiente - SEDAM/RO e Instituto Brasileiro de do Meio Ambiente e Recursos Naturais - IBAMA.

\section{Legislação de pesca}

As portarias que normatizam o "Período de Defeso" (período de proibição da pesca de determinadas espécies em função de estarem em período de reprodução) na região do Vale do Guaporé, utilizadas para a discussão foram: Portaria IBAMAN $N^{\circ} .65$ e 67 de 30 de outubro de 2003, Instrução Normativa (IN) do Ministério do Meio Ambiente No. 18 de 14 de outubro de 2004 e a Portaria SEDAM No 244 de 15 de dezembro de 2003. Essas portarias foram obtidas junto aos órgãos competentes (Instituto Brasileiro do Meio Ambiente e dos Recursos Naturais - IBAMA e Secretaria de Desenvolvimento Ambiental do Estado de Rondônia - SEDAM).

As tabelas apresentadas para as análises sintetizam o período do defeso estipulado na legislação citada.
No entanto, há diferenças entre elas quanto às espécies consideradas proibidas e também ao início e término do defeso, a IN N ${ }^{\text {o }} 18$ com início em 15 novembro e término em 15 março, enquanto as demais iniciam dia 01 de novembro e terminam 28 fevereiro.

\section{Resultados}

Verificou-se que as 28 categorias de pescado analisadas correspondem, no mínimo, a 32 gêneros taxonômicos distintos, o que indica o agrupamento de uma ou mais espécies com o mesmo nome popular atribuído pelos pescadores (Tabela 2). Das 28 categorias analisadas, 18 tiveram o seu período reprodutivo relatado pela maioria dos pescadores entrevistados (entre 70 e $80 \%$ dos pescadores) e para as demais (10), a informação foi relatada por um percentual menor de pescadores (30 a $60 \%$ ) (Tabela 1). Observa-se que no primeiro caso aparecem principalmente as espécies mais capturadas na pesca comercial local. As pescarias experimentais capturaram $14(50 \%)$ das categorias de pescado identificadas.

TABELA 1. Percentual de pescadores que informaram o período reprodutivo por categoria de pescado.

\begin{tabular}{c|l}
\hline $\begin{array}{c}\text { Percentual de } \\
\text { pescadores }\end{array}$ & \multicolumn{1}{c}{ Categorias de pescado } \\
\hline $80 \%$ & $\begin{array}{l}\text { caparari, cará, curimatá, flexeira, jaraqui, } \\
\text { peixe cachorro, piau, piranhas, pirarara. } \\
\text { surubim, tambaqui, traíra }\end{array}$ \\
\hline $70 \%$ & $\begin{array}{l}\text { branquinhas, pacu, pescada, sardinhas, } \\
\text { apapá, tucunaré }\end{array}$ \\
\hline $60 \%$ & cangati, mandubé \\
\hline 50 a $30 \%$ & $\begin{array}{l}\text { mandi, jatuarana, bacu, dourada, bico de } \\
\text { pato, filhote, cuiu cuiu, jaú }\end{array}$ \\
\hline
\end{tabular}


TABELA 2: Período reprodutivo das categorias de pescado comercializadas no mercado de Costa Marques (Rondônia), resultado do conhecimento ecológico tradicional dos pescadores locais; principais pontos de conflitos destacados pelos pescadores quanto a efetiva proteção do período reprodutivo pela portaria do defeso e o período de proteção de acordo com a legislação de Defeso (Portaria IBAMA № 65 e 67 de 30 de outubro de 2003, Instrução Normativa do Ministério do Meio Ambiente № 18 de 14 de outubro de 2004 e Portaria SEDAM № 244 de 15 de dezembro de 2003).

\begin{tabular}{|l|l|}
\hline$\Delta \boldsymbol{\Delta}$ & Período reprodutivo conforme conhecimento tradicional dos pescadores CET \\
\cline { 2 - 2 } & Principais pontos de conflitos pescadores versus portaria do defeso \\
\hline & Período de descanso reprodutivo, não citado como reprodutivo \\
\hline
\end{tabular}

\begin{tabular}{|c|c|c|c|c|c|c|c|c|c|c|c|c|}
\hline \multirow{3}{*}{\begin{tabular}{l}
\multicolumn{1}{c}{ Categorias } \\
Acarás \\
Astronotus spp.; Satanoperca spp.
\end{tabular}} & jan. & fev. & \multirow[t]{2}{*}{ mar. } & \multirow[t]{2}{*}{ abr. } & \multirow[t]{2}{*}{ maio } & \multirow[t]{2}{*}{ jun. } & \multirow[t]{2}{*}{ jul. } & \multirow[t]{2}{*}{ ago. } & \multirow[t]{2}{*}{ set. } & \multirow[t]{2}{*}{ out. } & \multirow{2}{*}{\multicolumn{2}{|c|}{\begin{tabular}{c|c} 
nov. & dez. \\
Defeso
\end{tabular}}} \\
\hline & \multicolumn{2}{|c|}{ Defeso } & & & & & & & & & & \\
\hline & $\Delta \boldsymbol{\Delta}$ & $\Delta \Delta$ & & & & & & & & & $\Delta \Delta$ & $\Delta \Delta$ \\
\hline $\begin{array}{l}\text { Apapás } \\
\text { Pellona spp. }\end{array}$ & $\boldsymbol{\Delta} \boldsymbol{\Delta}$ & & & & & & & & & & $\Delta \Delta$ & $\Delta \Delta$ \\
\hline $\begin{array}{l}\text { Aracus } \\
\text { Schizodon spp. } \\
\text { Leporinus spp. } \\
\text { Laemolyta spp. }\end{array}$ & $\Delta \boldsymbol{\Delta}$ & $\Delta \Delta$ & & & & & & & & & $\boldsymbol{\Delta} \boldsymbol{\Delta}$ & $\Delta \boldsymbol{\Delta}$ \\
\hline $\begin{array}{l}\text { Bacu } \\
\text { Hypostomus spp. }\end{array}$ & & & & & & & & & & & & $\Delta \Delta$ \\
\hline $\begin{array}{l}\text { Bico de Pato } \\
\text { Sorubim spp. }\end{array}$ & $\boldsymbol{\Delta} \boldsymbol{\Delta}$ & $\Delta \Delta$ & & & & & & & & & $\Delta \Delta$ & $\Delta \Delta$ \\
\hline $\begin{array}{l}\text { Branquinhas } \\
\text { Psectrogaster spp. } \\
\text { Potamorhina } \text { spp. }\end{array}$ & $\Delta \boldsymbol{\Delta}$ & & & & & & & & & & $\Delta \boldsymbol{\Delta}$ & $\Delta \Delta$ \\
\hline $\begin{array}{l}\text { Cangati } \\
\text { Parauchenipterus galeatus }\end{array}$ & $\Delta \boldsymbol{\Delta}$ & $\Delta \Delta$ & & & & & & & & & $\Delta \boldsymbol{\Delta}$ & $\Delta \Delta$ \\
\hline $\begin{array}{l}\text { Caparari* } \\
\text { Pseudoplatystoma tigrinum }\end{array}$ & $\boldsymbol{\Delta} \boldsymbol{\Delta}$ & $\Delta \boldsymbol{\Delta}$ & $\Delta \Delta$ & & & & & & & & & \\
\hline $\begin{array}{l}\text { Cuiu cuiu* } \\
\text { Oxydora niger }\end{array}$ & & & & & & & & & & & & $\Delta \Delta$ \\
\hline $\begin{array}{l}\text { Curimatã } \\
\text { Prochilodus nigricans }\end{array}$ & $\boldsymbol{\Delta} \Delta$ & $\Delta \Delta$ & & & & & & & & & & \\
\hline $\begin{array}{l}\text { Dourada } \\
\text { Branchyplatystoma rousseauxii }\end{array}$ & & & & & & & & & & & & $\Delta \Delta$ \\
\hline $\begin{array}{l}\text { Filhote } \\
\text { Branchyplatystoma filamentosum }\end{array}$ & & & & & & & & & & & & $\Delta \Delta$ \\
\hline $\begin{array}{l}\text { Flexeiras* } \\
\text { Hemiodus spp. }\end{array}$ & $\Delta \boldsymbol{\Delta}$ & & & & & & & & & & $\Delta \Delta$ & $\Delta \Delta$ \\
\hline $\begin{array}{l}\text { Jaraqui } \\
\text { Semaprochilodus spp }\end{array}$ & $\Delta \boldsymbol{\Delta}$ & $\Delta \boldsymbol{\Delta}$ & & & & & & & & & & \\
\hline $\begin{array}{l}\text { Jatuarana* } \\
\text { Brycon spp. }\end{array}$ & $\boldsymbol{\Delta} \mathbf{\Delta}$ & $\Delta \boldsymbol{\Delta}$ & & & & & & & & & & \\
\hline $\begin{array}{l}\text { Jaú* } \\
\text { Zungaro zungaro }\end{array}$ & & & & & & & & & & & & $\Delta \Delta$ \\
\hline $\begin{array}{l}\text { Mandi longo } \\
\text { Pimelodus spp. }\end{array}$ & $\Delta \Delta$ & & & & & & & & & & & \\
\hline $\begin{array}{l}\text { Mandubé } \\
\text { Ageneiosus brevifilis }\end{array}$ & & $\Delta \Delta$ & & & & & & & & & $\Delta \Delta$ & \\
\hline $\begin{array}{l}\text { Pacu* } \\
\text { Mylossoma spp. }\end{array}$ & $\boldsymbol{\Delta} \Delta$ & $\Delta \Delta$ & & & & & & & & & & $\Delta \Delta$ \\
\hline $\begin{array}{l}\text { Peixe cachorro } \\
\text { Acestrorhynchus spp. }\end{array}$ & $\Delta \boldsymbol{\Delta}$ & $\Delta \Delta$ & $\Delta \boldsymbol{\Delta}$ & & & & & & & & & \\
\hline
\end{tabular}




\begin{tabular}{|c|c|c|c|c|c|c|c|c|c|c|}
\hline $\begin{array}{l}\text { Pescada* } \\
\text { Plagioscion spp. }\end{array}$ & & & & $\Delta \Delta$ & $\Delta \Delta$ & & & & & \\
\hline $\begin{array}{l}\text { Piranhas } \\
\text { Serrassalmus spp. }\end{array}$ & $\Delta \Delta$ & & & & & & & & & $\Delta \Delta$ \\
\hline $\begin{array}{l}\text { Pirarara } \\
\text { Phractocephalus hemiliopterus }\end{array}$ & & & & & & & & $\Delta \Delta$ & $\Delta \Delta$ & $\Delta \Delta$ \\
\hline $\begin{array}{l}\text { Sardinhas* } \\
\text { Triportheus spp. }\end{array}$ & $\Delta \Delta$ & & & & & & & & $\Delta \Delta$ & $\Delta \Delta$ \\
\hline $\begin{array}{l}\text { Surubim* } \\
\text { Pseudoplatystoma fasciatum }\end{array}$ & $\Delta \Delta$ & $\Delta \mathbf{\Delta}$ & $\Delta \Delta$ & & & & & & & \\
\hline $\begin{array}{l}\text { Tambaqui* } \\
\text { Colossoma macropomum }\end{array}$ & & & & & & $\Delta \Delta$ & $\Delta \Delta$ & $\Delta \Delta$ & $\Delta \Delta$ & $\Delta \Delta$ \\
\hline $\begin{array}{l}\text { Traíra } \\
\text { Hoplias malabaricus }\end{array}$ & $\Delta \Delta$ & & & & & & & & $\Delta \Delta$ & $\Delta \Delta$ \\
\hline $\begin{array}{l}\text { Tucunaré* } \\
\text { Cichla spp. }\end{array}$ & & & & & & & & $\Delta \Delta$ & $\Delta \Delta$ & $\Delta \Delta$ \\
\hline
\end{tabular}

${ }^{*}$ Categorias com captura proibida durante o defeso.

A partir da comparação do conhecimento ecológico tradicional dos pescadores com a legislação de defeso, foram identificadas divergências quanto ao período reprodutivo para (7) categorias: caparari, tambaqui, surubim, pescada, pirarara, tucunaré e peixe cachorro, sendo as seis (6) primeiras indicadas como ponto de conflito entre o conhecimento tradicional e o período de defeso (Tabela 2). As demais categorias (21) na percepção dos pescadores têm o seu período reprodutivo protegido nos meses estipulados para o defeso nas referidas portarias.

O resultado obtido na comparação do conhecimento ecológico tradicional dos pescadores locais com os resultados obtidos nas pescarias experimentais (Tabela 3) demonstrou: 1) o CET confere tanto com a informação obtida experimentalmente quanto ao período de defeso para 10 categorias: aracus, branquinha, cangati, flexeiras, mandubé, pacu, peixe-cachorro, pescada, piranhas e traíra; 2) o CET sobre o período reprodutivo da espécie corresponde ao período do defeso, porém não é confirmada pelo resultado experimental em 4 categorias: tucunaré, sardinhas, bico de pato e curimatã. A informação do CET sobre o período reprodutivo não corrobora com o período do defeso em 6 categorias: caparari, pescada, surubim, tambaqui e tucunaré (Tabelas 2 e 3 ). Dentre essas categorias, apenas a pescada e o tucunaré puderam ser confirmadas pelo resultado experimental, indicando que o período reprodutivo confere com o CET e é diferente do período determinado pela legislação do defeso (Tabela 3 ).
O número de informações obtidas com o CET confirmadas experimentalmente, (10, ou seja, $71,5 \%$ das 14 espécies avaliadas) reforça a afirmativa de que o pescador possui grande conhecimento sobre a biologia das categorias de peixes que ele explora. No entanto, algumas afirmativas não puderam ser confirmadas experimentalmente, em função da ausência de dados suficientes sobre a reprodução das espécies que compõe algumas categorias.

\section{Discussão}

As comunidades amazônicas são reconhecidas pelo apurado conhecimento tradicional acumulado durante várias gerações no que diz respeito à região ou ambiente em que vivem e sobre as espécies de animais e vegetais por elas exploradas. Esse conhecimento foi construído em função da necessidade de sobreviver da floresta e dela retirar os produtos que geram recursos financeiros. Nas pescarias, o convívio diário do ribeirinho com o ambiente aquático somado a necessidade de exploração, lapidou a experiência do pescador o qual é capaz de reconhecer com eficiência a distribuição das espécies exploradas, as variações sazonais na abundância e os movimentos migratórios.

O uso do CET pode contribuir na pesquisa ecológica a partir da taxonomia e sistemática popular, que considera o meio no qual as diferentes culturas agrupam e caracterizam os organismos no seu entorno e pode ser um indicativo da diversidade local (Berkers et al., 2006). 
TABELA 3: Resultado obtido para o período reprodutivo das categorias de pescado comercializadas no mercado de Costa Marques, Rondônia, de acordo com o conhecimento ecológico tradicional (CET) pescadores locais, comparados ao período reprodutivo observados nas pescarias experimentais e com a portaria de defeso; Freqüência Reprodutiva (FR) de cada categoria.

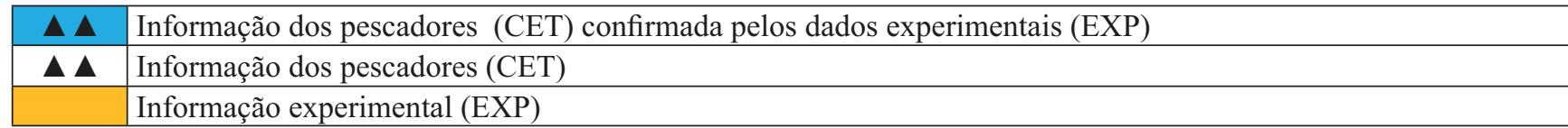

\begin{tabular}{|c|c|c|c|c|c|c|c|c|c|c|c|c|c|c|}
\hline \multirow{2}{*}{ CATEGORIAS } & & jan & fev & mar & abr & mai & jun & jul & ago & set & out & nov & dez & FR \\
\hline & & \multicolumn{2}{|c|}{ Defeso } & & & & & & & & & \multicolumn{2}{|c|}{ Defeso } & $\%$ \\
\hline \multirow{2}{*}{ Aracus } & CET & $\Delta \Delta$ & $\Delta \boldsymbol{\Delta}$ & & & & & & & & & $\Delta \boldsymbol{\Delta}$ & $\Delta \boldsymbol{\Delta}$ & \\
\hline & EXP & & & & & & & & & & & & & 43,08 \\
\hline \multirow{2}{*}{ Bico de Pato } & CET & $\Delta \boldsymbol{\Delta}$ & $\Delta \boldsymbol{\Delta}$ & & & & & & & & & $\Delta \boldsymbol{\Delta}$ & $\Delta \boldsymbol{\Delta}$ & \\
\hline & EXP & & & & & & & & & & & & & 38,10 \\
\hline \multirow{2}{*}{ Branquinhas } & CET & $\Delta \boldsymbol{\Delta}$ & & & & & & & & & & $\Delta \Delta$ & $\Delta \boldsymbol{\Delta}$ & \\
\hline & EXP & & & & & & & & & & & & & 43,24 \\
\hline \multirow{2}{*}{ Cangati } & CET & $\Delta \Delta$ & $\Delta \Delta$ & & & & & & & & & $\Delta \boldsymbol{\Delta}$ & $\Delta \boldsymbol{\Delta}$ & \\
\hline & EXP & & & & & & & & & & & & & 47,06 \\
\hline \multirow{2}{*}{ Curimatã } & CET & $\Delta \boldsymbol{\Delta}$ & $\Delta \boldsymbol{\Delta}$ & & & & & & & & & & & \\
\hline & EXP & & & & & & & & & & & & & 31,3 \\
\hline \multirow{2}{*}{ Flexeiras } & CET & $\Delta \Delta$ & & & & & & & & & & $\Delta \Delta$ & $\Delta \Delta$ & \\
\hline & EXP & & & & & & & & & & & & & 7,95 \\
\hline \multirow{2}{*}{ Mandubé } & CET & & $\Delta \boldsymbol{\Delta}$ & & & & & & & & & $\Delta \Delta$ & & \\
\hline & EXP & & & & & & & & & & & & & 1,01 \\
\hline \multirow{2}{*}{ Pacu } & CET & $\Delta \Delta$ & $\Delta \boldsymbol{\Delta}$ & & & & & & & & & & $\Delta \boldsymbol{\Delta}$ & \\
\hline & EXP & & & & & & & & & & & & & 1,16 \\
\hline \multirow{2}{*}{ Peixe cachorro } & CET & $\Delta \Delta$ & $\Delta \boldsymbol{\Delta}$ & $\Delta \boldsymbol{\Delta}$ & & & & & & & & & & \\
\hline & EXP & & & & & & & & & & & & & 15,12 \\
\hline \multirow{2}{*}{ Pescada } & CET & & & & $\Delta \boldsymbol{\Delta}$ & $\Delta \mathbf{\Delta}$ & & & & & & & & \\
\hline & EXP & & & & & & & & & & & & & 22,22 \\
\hline \multirow{2}{*}{ Piranhas } & CET & $\Delta \Delta$ & & & & & & & & & & & $\Delta \Delta$ & \\
\hline & EXP & & & & & & & & & & & & & 31,25 \\
\hline \multirow{2}{*}{ Sardinhas } & CET & $\Delta \boldsymbol{\Delta}$ & & & & & & & & & & $\Delta \boldsymbol{\Delta}$ & $\Delta \boldsymbol{\Delta}$ & \\
\hline & EXP & & & & & & & & & & & & & 40,00 \\
\hline \multirow{2}{*}{ Traíra } & CET & $\Delta \boldsymbol{\Delta}$ & & & & & & & & & & $\Delta \Delta$ & $\Delta \boldsymbol{\Delta}$ & \\
\hline & EXP & & & & & & & & & & & & & 17,4 \\
\hline \multirow{2}{*}{ Tucunaré } & CET & & & & & & & & & & $\Delta \boldsymbol{\Delta}$ & $\Delta \boldsymbol{\Delta}$ & $\Delta \boldsymbol{\Delta}$ & \\
\hline & EXP & & & & & & & & & & & & & 28,57 \\
\hline
\end{tabular}

Dentro do escopo da taxonomia popular os resultados do presente trabalho demonstraram que o conhecimento tradicional dos pescadores pode incluir mais de uma espécie da ictiofauna em cada categoria de pescado identificada. Algumas, como é o caso dos aracus e pacus, incluem mais de dois gêneros em uma mesma categoria de pescado. Esses agrupamentos são reconhecidos na literatura e recebem as denominações de etnoespécies ou etnogêneros e são inseridos em etnofamílias (CostaNeto et al., 2002; Ramires et al., 2007).

As análises do conhecimento dos pescadores relativos às etnofamílias, compõem um sistema de informações biológicas e ecológicas úteis para gestão da pesca de pequena escala, especialmente nos trópicos, onde há uma alta diversidade de espécies e um baixo conhecimento ecológico e biológico específico (Drew, 
2005; Silvano et al., 2006; Ramires et al., 2007). Situação esta na qual podem ser inclusas áreas pouco citadas na literatura como em território brasileiro nas bacias do Guaporé e Mamoré.

Este refinado conhecimento sobre as categorias é confirmado na alta porcentagem de pescadores que souberam indicar o período reprodutivo da maioria das espécies. Cerca de 70 a $80 \%$ dos pescadores identificaram o período reprodutivo da maioria das categorias analisadas (65\%), principalmente das espécies comerciais com as quais têm mais contato. As demais espécies (35\%) tiveram o período reprodutivo identificado por um menor número de pescadores, provavelmente pela baixa representação econômica na pesca. Uma explicação para a ausência de conhecimento dos pescadores sobre a reprodução de algumas espécies de peixes se deve ao fato dos eventos reprodutivos serem curtos e difíceis de serem observados, ao contrário de outros aspectos biológicos como habitat e dieta (Silvano e Begossi, 2002; Silvano et al., 2006). Além disso, algumas espécies os pescadores tem menos contato em função da baixa representatividade na pesca profissional e por isso não conseguem identificar seu período reprodutivo (Silvano et al., 2006).

Este CET dos pescadores sobre as espécies comerciais permite inferências e questionamentos sobre a efetividade das normas de proteção e manejo do pescado adotadas nessas localidades. Durante os anos de 2003 e 2004, nas discussões sobre o ordenamento pesqueiro da região, os pescadores freqüentemente chamavam a atenção para as falhas em relação ao período de defeso estabelecido. As principais espécies comerciais dos mercados pesqueiros do Guaporé-Mamoré (especialmente caparari, tambaqui, surubim, pescada e tucunaré) eram apontadas como categorias desprotegidas pela lei do defeso naquela região (Doria et al, 2004). Na percepção dos pescadores locais havia uma diferença entre o período reprodutivo observado por eles para essas categorias em relação ao período estabelecido para o defeso reprodutivo. A base de dados biológicos utilizada para a elaboração das portarias de defeso no estado de Rondônia não considerou possíveis peculiaridades para cada sub-bacia (C. R. C. Doria, observação pessoal), desconsiderando a Lei Estadual $\mathrm{n}^{\circ} 1038$ de 22 de janeiro de 2002 que no seu artigo $5^{\circ}$ afirma: "A proibição à pesca deverá ser adotada com base em estudos científicos, voltados à determinação de parâmetros biológicos e biométricos e de estatísticas indicadoras do volume populacional pesqueiro e nível de captura máxima sustentável nos estoques existentes nas bacias hidrográficas".

As políticas de manejo são limitadas pela ausência de informações pontuais sobre a biologia, reprodução e tamanhos de primeira maturação sexual das espécies. Órgãos responsáveis pela implementação de medidas de ordenamento pesqueiro utilizam a forma convencional de gestão como o estabelecimento de épocas de defeso e regulação de tamanhos mínimos de captura e aparelhos de pesca, na maioria das vezes, sem a incorporação de informações científicas suficientes para subsidiar adequadamente o ordenamento pesqueiro. As normas de defeso e restrição do tamanho de captura regidas pela Portaria MMA/IBAMA No 8, de 2 de Fevereiro de 1996 e Portaria No 1534, de 20 de Dezembro de 1989 para a pesca de algumas espécies de peixes são emitidas anualmente para toda Amazônia brasileira, com raras exceções de regionalização entre bacias e sub-bacias hidrográficas (Batista et al., 2004). Conseqüentemente, desconsideram as variações regionais impostas pelas condições ambientais locais na biologia das populações de peixes.

$\mathrm{Na}$ década de 90, normas oriundas de outras localidades desconsideraram os efeitos ambientais locais na manutenção das populações de peixes da região de Santarém e o estabelecimento da lei de forma igualitária no defeso para a maioria dos Characiformes resultou em um ordenamento pesqueiro deficiente (IBAMA/Projeto IARA, 1993). Soma-se a este cenário, o fato de que os períodos de defeso, atualmente propostos pelos órgãos gestores, não abrangem os picos de desova de várias espécies que possuem atividade reprodutiva durante mais de um período ao longo do ano, como verificado por Paschoal et al. (2004), ao avaliar estratégias reprodutivos de alguns peixes do médio rio Xingu.

Estudos sobre a biologia pesqueira devem considerar e identificar no desembarque as diferentes espécies envolvidas em cada categoria de pescado, devido à peculiaridades distintas da biologia entre espécies (Torrente-Vilara, 2003) e as variações macroregionais no pulso de inundação (Goulding et al., 2003). Esta questão tem 
sido evidenciada em estudos como de: estrutura genética de surubins e capararis dos rios Madalena, Orinoco e Amazonas, onde foram verificadas diferenças significativas entre as populações de surubins das três bacias e para o caparari entre o Orinoco e Amazonas (Ramirez, 2001); e sobre estudos de crescimento de Plagioscium squamosissimus, onde variações foram obtidas em dados provenientes de lagos distintos da Amazônia central (Wothamann, 1979).

Os conflitos levantados pelos pescadores de Costa Marques podem ser fundamentados na diferença existente entre aspectos da biologia das espécies desta sub-bacia, em relação às demais sub-bacias amazônicas, fornecedoras da base de dados para a elaboração da portarias de defeso. De acordo com a percepção dos pescadores as principais espécies comerciais dos mercados pesqueiros do Guaporé-Mamoré (caparari, tambaqui, surubim, pescada e tucunaré) estão desprotegidas pela lei do defeso. Neste estudo, foi observado que o caparari, o tambaqui e a pescada reproduzem-se em período distinto do determinado para o defeso. Para o surubim e caparari, cuja atividade reprodutiva aparentemente concentrada de janeiro a março, somente parte do período reprodutivo estaria efetivamente protegida pela portarias em estudo (novembro a fevereiro). A pescada e o tucunaré tiveram as informações obtidas com os pescadores confirmadas nas pescarias experimentais, indicando que o período de maior atividade reprodutiva não confere com a legislação discutida.

De acordo com os pescadores, o caparari e o surubim (Tabela 2) se reproduzem no período de janeiro a março. Trabalhos realizados na região da bacia do Mamoré (adjacente ao Guaporé) verificaram que: para o surubim Pseudoplatystoma fasciatum, o período determinado foi janeiro e fevereiro (na $1^{\mathrm{a}}$ fase da enchente) (Loubens e Panfili, 2000). Essas informações sobre o surubim confirmam as obtidas a partir do conhecimento ecológico tradicional e também com o período para o "defeso". Contudo, considerando os resultados obtidos por Loubens (2003) para a pescada Plagioscium squamosissimus, com dois picos de atividade reprodutiva sendo um $\left(1^{\circ}\right)$ em setembro-outubro e outro $\left(2^{\circ}\right)$ em junho-julho, o defeso não estaria incluindo todo período reprodutivo. Neste trabalho, de acordo com a percepção dos pescadores a atividade reprodutiva de P. squamosissimus ocorre nos meses de abril e maio e as pescarias experimentais capturaram exemplares em reprodução em maio. Essa diferença antecipou em 1-2 meses a fase de maior atividade reprodutiva quando comparado ao resultado obtido por Loubens (2003).

O período reprodutivo estimado para tambaqui, Colossoma macropomum, pelos pescadores da região é de agosto a outubro. Na região de Trinidad (bacia do Mamoré) foi observado que essa espécie apresenta dois picos reprodutivos: um em outubro e outro em dezembro (Loubens e Panfili, 1997; Villacorta-Corrêa, 1997). Ao considerar o conhecimento ecológico tradicional dos pescadores sobre a reprodução do tambaqui nesse estudo, verificamos também uma antecipação do período de maior atividade reprodutiva, de agosto a outubro (Tabela 2). Dois dos três meses de atividade reprodutiva estão fora do período estipulado para o defeso e aparentemente, para o tambaqui, a portaria citada não atinge seus objetivos de proteção do período reprodutivo na sub-bacia do Guaporé.

Diferenças representadas pela antecipação da maior atividade reprodutiva de espécies com desova total poderiam incluir respostas biológicas às variações interanuais na reprodução entre diferentes regiões, visto que há um sincronismo entre as fases hidrológicas para assegurar a sobrevivência da prole nas áreas inundadas durante os períodos de enchente-cheia (Araújo-Lima, 1990; Lowe-McConnell, 1999). Essas peculiaridades não podem ser desconsideradas na elaboração das políticas de manejo.

As informações dos pescadores também corroboram com informações obtidas por outros pesquisadores. O período reprodutivo do Curimatã Prochilodus nigricans observado em janeiro e fevereiro (Tabela 2), sobrepõe, em parte, o período observado para essa espécie na região de Trinidad, dezembro e janeiro por Loubens e Panfili (2000). Para os aracus, o período de novembro a fevereiro, coincide totalmente com o observado para Schizodon fasciatum por Isaac e Cerdeira, (2004) no médio Amazonas. Para as branquinhas, o período de novembro a janeiro, coincide com o observado por Junk (1985), entre dezembro a março. Para o pacu o período de dezembro a fevereiro coincide com o observado para 
Metynnis spp e Mylossoma spp de dezembro a março por Junk (1985). Para as sardinhas, o período de novembro a janeiro, coincide com o observado no rio Madeira, entre dezembro a fevereiro para Triportheus albus (Dados do Laboratório e Ictiologia e Pesca/ UNIR não publicados) e para T. elongatus de dezembro a março por Junk (1985). O tucunaré teve o período reprodutivo experimental determinado em setembro; o observado pelo CET, entre outubro e dezembro, coincide parcialmente com o observado por Junk (1985) de dezembro a julho, porém difere do observado por Isaac e Cerdeira (2004) no baixo Amazonas, período reprodutivo de março a abril. As divergências observadas para a ordem Perciformes, como é o caso das categorias da família Cichlidae (acáras e tucunarés) e Scianidae (pescadas), podem ser efeito da estratégia de desova parcelada dessas espécies (Chellapa et al., 2003) ocasionando diferenças na reprodução entre as diferentes sub-bacias.

Deve ser ressaltado que as categorias dos aracus, branquinhas, sardinhas e pacu são representadas por diversas espécies pertencentes a mais de um gênero (Tabela 2) e podem ocorrer peculiaridades interespecíficas sobre a biologia reprodutiva que não puderam ser consideradas nesse trabalho. Aparentemente, o ciclo de vida da maioria das espécies que compõe essas categorias é fortemente regida pelo pulso de inundação e assim, a reprodução deve ocorrer, para a maioria delas, na enchente-cheia como ocorre para uma grande parte dos Characiformes (Junk, 1985; Vazzoler e Menezes, 1992; Merona e Gascuel, 1993; Lowe-McConnell, 1999), sendo que o início da enchente na região ocorre em novembro. De qualquer forma, categorias que envolvem mais de uma espécie necessitam de estudos específicos para esclarecer detalhes biológicos sobre a reprodução.

O gênero Cichla também envolve mais de duas espécies (Kullander e Ferreira, 2006). E somado ao fato de apresentam desova parcelada (Chellappa et al., 2003) com a ausência de informações experimentais nesse trabalho para determinação do período reprodutivo, dificultou conclusões sobre essa categoria. De qualquer forma, é pouco provável que o período de defeso, de novembro a fevereiro, seja realmente efetivo tendo em vista que a espécie apresenta dois picos importantes de desova: um em outubro e outro em abril (dados obtidos em reservatório, Chellappa et al., 2003).

A elevada concordância constatada entre o conhecimento ecológico tradicional e o conhecimento científico reforça as afirmações de que o CET pode ser utilizado para a compreensão da dinâmica ecológica de ecossistemas e subsidiar formas alternativas de gestão baseada nos ecossistemas ${ }^{3}$ (incluindo os seres humanos neste sistema) e na participação das populações locais (Bekers et al. 2006).

As duas metodologias de geração de conhecimento sobre os recursos naturais locais, o científico e o tradicional, se mostram complementares na construção das novas formas de gestão. Procedimentos como esses somados a efetiva participação dos diversos atores podem contribuir com o sucesso dos planos de gestão (Bekers et al., 2006).

A partir deste trabalho foi possível concluir que:

1. O conhecimento ecológico tradicional do pescador local é forte e informativo para gerar informações básicas sobre a biologia reprodutiva das categorias de pescado locais, permitindo adequar a política da pesca naquele trecho do rio Guaporé.

2. Os resultados do presente estudo permitem sugerir que a portaria direcionada à bacia do rio Guaporé (Rondônia) seja revista e específica para cada categoria, considerando: a) caparari e surubim: estender o defeso para o mês de março; b) pescada: estabelecer o defeso entre abril e maio; c) tambaqui: antecipar o defeso para o mês de agosto e mantê-lo até fevereiro; d) tucunaré: estabelecer o defeso entre setembro e fevereiro.

\footnotetext{
3 Um dos aspectos característicos da gestão baseada em ecossistema é a sua ênfase em proteger o potencial produtivo do sistema que produz fluxos de recursos, em vez de proteger uma espécie ou estoque individual como recurso (Bekers et al., 2006).
} 


\section{Referências}

Allegretti, M. H. 1987. Reservas Extrativistas: uma proposta de desenvolvimento para a floresta Amazônica. EIA, Curitiba, Brasil, 77pp.

Ankei, Y. 1982. Folk-knowledge of the fish among the Songola and the Bwari: comparative ethnoichthyology of the Zaire River and Lake Tanganyica fishermen. Africa Kenkyu, 21: 1-56.

Araújo-Lima C. A. R. M. 1990. Larval development and reproductive strategies of central Amazon fishes. $\mathrm{PhD}$ Thesis, University of Stirling, Scotland, UK, 155pp.

Azevedo, C. R de; Apel, M. 2004. Co-gestão: Um processo em construção na várzea amazônica. Estado Pará - Estudo Estratégico - Analítico. IBAMA/ProVárzea, Manaus, Brasil, 100pp.

Batista, V. S. 1998. Distribuição, dinâmica da frota e dos recursos pesqueiros da Amazônia Central. Tese de Doutorado, Instituto Nacional de Pesquisas da Amazônia/Fundação Universidade Federal do Amazonas, Brasil, 291pp.

Batista V. S.; Isaac V. J.; Viana, J. P. 2004. Exploração e manejo dos recursos pesqueiros da Amazônia. In: Ruffino, M. L. (coord.). A pesca e os recursos pesqueiros na Amazônia Brasileira. IBAMA/ProVárzea, Manaus, Brasil, p.63- 151.

Batistella, A. M; Castro; C. P; Vale; J. D. 2005. Conhecimento dos moradores da comunidade de Boas Novas, no lago Januacá - Amazonas, sobre os hábitos alimentares dos peixes da região. Acta Amazonica, 35 (1): 51-54.

Begossi, A.; Garavello, J. C. 1990. Notes on the etnoicthyology from Tocantins River. Acta Amazonica, 20: 341-351

Begossi, A.; Figueiredo, J. L. 1995. Ethnoichtthyoloy of southern coastal fishermem: cases from Búzios Island and Sepetiba Bay (Brazil). Bull Mar Science, 56: 710-717

Berkers, F.; Mahon, R.; McConney, P.; Pollnac, R.; Pomery, R. (autores da versão original em inglês). Kaliskoski, D. C. (org. edição em português). 2006. Gestão de pesca de pequena escala: diretrizes e métodos alternativos. Ed. FURG, Rio Grande, Brasil. 360pp.

Castro, E. 2000. Território, biodiversidade e saberes de populações tradicionais. In: Diegues, A. C. (org.). Etnoconservação. Novos rumos para a conservação da natureza. HUCITEC, NUPAUBUSP, São Paulo, Brasil, p.166-177.

Costa-Neto, E. M; Dias, C. V; Melo, M. N. 2002. O conhecimento ictiológico tradicional dos pescadores da cidade de Barra, região do médio São Francisco, Estado da Bahia, Brasil. Acta Scientiarum, 24 (2): 561-572.

Chellappa, S.; Câmara, M. R.; Chellappa, N. T. ; Beveridge, M. C. M ; Huntingford, F. A. 2003. Reprodutive ecology of a neotropical ciclhid fish, Cichla monoculus (Osteichytes: Cichlidae). Brazilian Journal Biology, 63 (1): 17-26.

Diegues, A. C. 2000. Etnoconservação da natureza: Enfoques alternativos. In: Diegues, A. C. (org.). Etnoconservação. Novos rumos para a conservação da natureza. HUCITEC, NUPAUB-USP, São Paulo, Brasil, p.1-46.

Doria, C. R. C.; Borges, M.; Brasil de Souza, S. T.; Lopes, L. J. 2004. A pesca e o Turismo no vale do Guaporé-Mamoré: Bases para ordenamento. Série Técnica. ECOPORÉ/WWF - Brasil, Porto Velho, Brasil, 58 pp.
Drew, J. A. 2005. Use of traditional ecological knowledge in marine conservation. Conservation Biology, 19 (4): 1286 -1293.

Fernandes, L. C.; Guimarães, S. C. P. (orgs). 2002. Atlas Geoambiental de Rondônia. SEDAM/GOVERNO DE RONDÔNIA, Porto Velho, Brasil, 150pp.

Gouding, M. R.; Barthem, R.; Ferreira, E. 2003. The Smithsonian atlas of the Amazon. Smithsonian Books, Washimgton, USA, 253pp.

IBAMA/Projeto IARA. 1993. Subsídios técnicos para o ordenamento pesqueiro na região do Médio Amazonas. IBAMA, Santarém, Brasil, 20pp.

Isaac, V. J.; Cerdeira, R. G. P. 2004. Avaliação e monitoramento de impacto dos acordos de pesca. Região do Médio Amazonas. IBAMA/ProVárzea, Manaus, Brasil, 61pp.

Junk, W. J. 1985. Temporary fat storage, and adaptation of some fish species to the waterlevel fluctuations and related environmental changes of the Amazon river. Amazoniana, 9 (3): 315-351.

Junk, W. J.; Bayley, P. B.; Sparks, R. E. 1989. The flood pulse concept in river-floodplain systems. Proceedings of the International Large River Symposium, Canadian special Publication of Fisheries and Aquatic Sciences, 106: 110-127.

Kullander, S. O.; Ferreira, E. J. G. 2006 A review of the South American cichlid genus Cichla, with descriptions of nine new species (Teleostei: Cichlidae). Ichthyological Exploration Freshwaters, 17 (4): 289-398.

Loubens, G. 2003. Biologie de Plagioscium squamosissimus (Teleostei: Sciaenidae) dans le bassin du Mamoré (Amazonie bolivienne). Ichthyological Exploration Freshwaters, 14 (4): 335-352.

Loubens, G.; Panfili, J. 1997. Biologie de Colossoma macropomum (Teleostei: Serrasalmidae) dans lê bassin du Mamoré (Amazonie bolivienne). Ichthyological Exploration Freshwaters, 8: 1-22.

Loubens, G.; Panfili, J. 2000. Biologie de Pseudoplatystoma fasciatum e P. tigrinum (Teleostei: Pimelodidae) dans lê bassin du Mamoré (Amazonie bolivienne). Ichthyological Exploration Freshwaters, 11(1): 13-34.

Lowe-McConnel, R. H. 1999. Estudos ecológicos de comunidades de peixes tropicais. EDUSP, São Paulo, Brasil, 453 pp.

Marques, J. G. W. 1995. Etnoictiologia: pescando pescadores nas águas da transdisciplinaridade. Resumos do IV Encontro Brasileiro de Ictiologia, Campinas, Brasil, p.41.

McGrath, D. G.; Castro, F.; Futemma, C. 1994; Reservas do lago e o manejo comunitário da pesca no Baixo Amazonas: Uma avaliação preliminar. In: Furtado, L.; Mello, A. F. \& Leitão, W. (eds). Povos das águas realidade e perspectiva na Amazônia. MPEG/UFPA, Belém, Brasil, p.213-229.

Merona, B.; Gascuel, D. 1993. Effects of flood regime and fishing effort on the overall abundance of an exploited fish community in the Amazon floodplain. Aquatic Living Resources, 6 (1): 97-108. Morril, W. T. 1967. Ethnoichtyology of the Cha-Cha. Ethology, 4: 405-416.

Olmos, F.; Queiroz Filho, A. P.; Lisboa, C. A. 1998. Unidades de Conservação de Rondônia. SEPLAN/PLANAFLORO/PNUD, Porto Velho, Brasil, 94pp.

Paschoal, J. P.; Botelho, M. C.; Brito, S. A. C, Chaves, R. C. Q, Camargo, M.; Isaac, V. J. 2004. Hábitos reprodutivos de alguns peixes 
do médio Rio Xingu Amazônia Brasileira PA Resumos do XXV Congresso Brasileiro de Zoologia, Brasília, Brasil, p.56.

Ramires, M.; Molina, S. M. G.; Hanazaki, N. 2007. Etnoecologia caiçara: o conhecimento dos pescadores artesanais sobre aspectos ecológicos da pesca. Biotemas, 20 (1): 101-113.

Ramirez, H. 2001. Diferenciação genética de populações de surubim (Pseudoplatystoma fasciatus) e de caparari (Pseudoplatystoma tigrinun) nas bacias do Magdalena, Orinoco e Amazonas. Tese de Doutorado, Instituto Nacional de Pesquisa da Amazônia/Fundação Universidade Federal do Amazonas, Manaus, Brasil 114pp.

Silvano, R. A. M.; Begossi, A. 2002. Ethnoichthyology and fish conservation in the Piracicaba river (Brazil). Journal of Ethnobiology, 22 (2): 285-306.

Silvano, R. A. M.; MacCord, P. F. L. ; Lima; R. V.; Begossi, A. 2006. When does this fish spawn? Fisheremen's local knowledge of migration and reproduction of Brazilian coastal fihes. Envioronmental Biolgy of Fish, 76: 371-386.

SNUC - Sistema Nacional de Unidades de Conservação da Natureza. 2002. Lei no. 9985, de 18 de julho de 2000; Decreto no. 4340, de agosto de 2002. $2^{\mathrm{a}}$ ed. MMA/SBF, Brasília, Brasil, 52pp.

Smith, N. H. 1979. A pesca no Rio Amazonas. INPA/CNPq, Manaus/Brasília, Brasil, 154pp.
Torrente-Vilara, G. 2003. Padrões nos parâmetros biológicos de três espécies de aracus (Characiformes: Anostomidae) em lagos de várzea da Amazônia Central e suas relações com a variação espaço-temporal. Dissertação de Mestrado, Instituto Nacional de Pesquisas da Amazônia/Fundação Universidade Federal do Amazonas, Manaus, Brasil, 90pp.

Vazzoler, A. E. A. M. 1996. Biologia da reprodução de peixes teleósteos: teoria e prática. EDUEM, Maringá, Brasil, 169pp.

Vazzoler, A. E. A. M.; Menezes, N. A. 1992. Síntese de conhecimentos sobre o comportamento reprodutivo dos Characiformes da América do Sul (Teleostei, Ostariophysi). Revista Brasileira de Biologia, 52 (4): 627-640.

Villacorta-Corrêa, M.A. 1997. Estudos de idade e crescimento do tambaqui Colossoma macropomum (Characiformes, Characidade) no Amazonas Central, pela analise de marcas sazonais e nas estruturas mineralizadas e microestruturas nos otólitos. Tese de Doutorado, Instituto Nacional de Pesquisa da Amazônia/ Fundação Universidade Federal do Amazonas, Brasil, 217pp.

Wothamann, H. O. W. 1979 A relação entre o desenvolvimento do otólito e o crescimento do peixe como auxílio na distinção de populações da pescada (Plasgioscion squamosissimus). Acta Amazônica, 9: 573-586. 